\title{
Critical behavior of the random-anisotropy model in the strong-anisotropy limit
}

\author{
Francesco Parisen Toldin, ${ }^{1,3}$ Andrea Pelissetto,${ }^{2}$ and Ettore \\ Vicari $^{3}$ \\ ${ }^{1}$ Scuola Normale Superiore, Pisa, Italy \\ 2 Dipartimento di Fisica dell'Università di Roma "La Sapienza" and INFN, Roma, \\ Italy. \\ ${ }^{3}$ Dipartimento di Fisica dell'Università di Pisa and INFN, Pisa, Italy. \\ E-mail: f.parisentoldin@sns.it, Andrea.Pelissetto@roma1.infn.it, \\ Ettore.Vicari@df.unipi.it
}




\begin{abstract}
We investigate the nature of the critical behavior of the randomanisotropy Heisenberg model (RAM), which describes a magnetic system with random uniaxial single-site anisotropy, such as some amorphous alloys of rare earths and transition metals. In particular, we consider the strong-anisotropy limit (SRAM), in which the Hamiltonian can be rewritten as the one of an Ising spin-glass model with correlated bond disorder. We perform Monte Carlo simulations of the SRAM on simple cubic lattices of linear size $L$, up to $L=30$, measuring correlation functions of the replica-replica overlap, which is the order parameter at a glass transition. The corresponding results show critical behavior and finite-size scaling. They provide evidence of a finite-temperature continuous transition with critical exponents $\eta_{o}=$ $-0.24(4)$ and $\nu_{o}=2.4(6)$. These results are close to the corresponding estimates that have been obtained in the usual Ising spin-glass model with uncorrelated bond disorder, suggesting that the two models belong to the same universality class. We also determine the leading correction-to-scaling exponent, finding $\omega=1.0(4)$.
\end{abstract}

PACS numbers: 75.50.Lk, 05.70.Jk, 75.40.Mg, 77.80.Bh

\title{
1. Introduction
}

The critical behavior of magnetic systems in the presence of quenched disorder has been the subject of extensive theoretical and experimental study. An important class of systems consists in amorphous alloys of rare earths with aspherical electron distributions and transition metals, for instance $\mathrm{TbFe}_{2}$ and $\mathrm{YFe}_{2}$. They are modeled [1] by a Heisenberg model with random uniaxial single-site anisotropy defined on a simple cubic lattice, or, in short, by the random-anisotropy model (RAM)

$$
\mathcal{H}=-J \sum_{\langle x y\rangle} \vec{s}_{x} \cdot \vec{s}_{y}-D \sum_{x}\left(\vec{u}_{x} \cdot \vec{s}_{x}\right)^{2}
$$

where $\vec{s}_{x}$ is a three-component spin variable, $\vec{u}_{x}$ is a unit vector describing the local (spatially uncorrelated) random anisotropy, and $D$ the anisotropy strength. In amorphous alloys the distribution of $\vec{u}_{x}$ is usually taken to be isotropic, since, in the absence of crystalline order, there is no preferred direction.

Random anisotropy is a relevant perturbation of the pure Heisenberg model, so that random-anisotropy systems show a critical behavior that is different from the Heisenberg one. For small $D$ however, Heisenberg behavior may still be observed, the Heisenberg fixed point controlling the multicritical behavior of the system. If $T_{p} \equiv T_{c}(D=0)$ is the critical temperature of the pure Heisenberg model, in the limit $t_{p} \equiv T / T_{p}-1 \rightarrow 0$ and $D \rightarrow 0$ the free energy has the scaling form 2

$$
\mathcal{F}=\left|t_{p}\right|^{2-\alpha_{H}} f\left(D^{2}\left|t_{p}\right|^{-\phi_{D}}\right),
$$

where $\alpha_{H}=-0.1336(15)$ is the specific-heat exponent in the pure Heisenberg theory [3, 4], $\phi_{D}=0.412(3)$ is the crossover exponent associated with the random-anisotropy perturbation [2], and $f(x)$ is a universal function. $\neq$

$\ddagger$ As a consequence of Eq. (2), for sufficiently small $D$ the critical-temperature shift is given by $T_{c}(D)-T_{c}(0) \approx c D^{2 / \phi_{D}}+a_{1} D^{2}+a_{2} D^{4}+\ldots$ where $2 / \phi_{D} \approx 4.9$. Note that the nonanalytic term 
The critical behavior in the presence of random anisotropy has been investigated at length, but a satisfactory picture has not been achieved yet. A recent review can be found in [5]. The Imry-Ma argument [6, 7] forbids the appearance of a low-temperature phase with nonvanishing magnetization for $d<4$. This is also supported by field-theoretical renormalization-group $(\mathrm{RG})$ calculations using the replica method [8, 9, 10, 2]. However, a glassy transition with a low-temperature phase characterized by quasi-long-range order (QLRO), i.e., a phase in which correlation functions decay algebraically, is still possible [7]. A Landau-Ginzburg calculation [1] of the equation of state for $D \rightarrow 0$ and a recent $4-\epsilon$ study [12, 13] based on a functional RG approach support this scenario in the weak-anisotropy limit. In the large-anisotropy limit $D \rightarrow \infty$ the model becomes an Ising spin glass with a correlated bond distribution. An interesting hypothesis, originally put forward in [14, is that in this limit the RAM transition is in the same universality class as that of the Ising spin-glass model (ISGM) [15, 16, 17.

Numerical simulations (see, for example, [18, 19, 20, 21, 22] ) provide some evidence of the existence of a finite-temperature transition for small values of $D$. On the other hand, for large $D$, even the presence of a finite-temperature transition is not supported by numerical simulations [22]. Experiments support the absence of a ferromagnetic phase for amorphous systems in general, and provide evidence of a generic glassy behavior at sufficiently low temperature. The nature of the transition and of the lowtemperature phase is however unclear. In particular, no evidence of QLRO has been reported.

The above-reported arguments apply to the RAM in which anisotropy has a generic cubic-symmetric distribution. However, in some particular cases, when disorder preserves the reflection symmetry $s_{x, a} \rightarrow-s_{x, a}, s_{x, b} \rightarrow s_{x, b}$ for $b \neq a$ (this is, for example, realized when the probability distribution vanishes outside the lattice axes), there is a standard order-disorder transition with a low-temperature magnetized phase. Moreover, a RG analysis of the corresponding Landau-Ginzburg-Wilson theory [2, 9, 23. shows that continuous transitions belong to the same universality class as that of the random-exchange Ising model (REIM). $\S$

In this paper we investigate the critical behavior of the RAM for a uniform distribution in the limit $D / J \rightarrow \infty$. In this case we can write $\vec{s}_{x}=\sigma_{x} \vec{u}_{x}$, with $\sigma_{x}= \pm 1$. Thus, the RAM reduces to a particular Ising spin-glass model with Hamiltonian [18]

$$
\mathcal{H}=-\sum_{\langle x y\rangle} j_{x y} \sigma_{x} \sigma_{y}, \quad j_{x y}=\vec{u}_{x} \cdot \vec{u}_{y},
$$

which we call strong random-anisotropy model (SRAM) (We set $J=1$ without loss of generality). Model (3) differs from the usual ISGM in the bond distribution. Here $D^{2 / \phi_{D}}$ is suppressed with respect to the first two analytic terms $D^{2}$ and $D^{4}$.

$\S$ The main difference with respect to the REIM critical behavior (see, e.g., 44 24]) is the approach to the asymptotic critical behavior, which is controlled by scaling corrections with exponent $\Delta=-\alpha_{r}$, where $\alpha_{r} \simeq-0.05$ is the specific-heat exponent of the REIM [2]. This is much smaller than the correction-to-scaling exponent of the REIM, which is $\Delta_{\text {REIM }} \approx 0.25$. 
the random variables $j_{x y}$ on different lattice links are correlated. For instance, one has $\overline{\prod_{\square} j_{x y}}=1 / 27$, where the product is over the links belonging to a given plaquette and the average is taken with respect to the distribution of the vectors $\vec{u}_{x}$. Thus, the SRAM is not only of interest as a model of a class of magnetic systems, but also per se, to understand how glassy behavior in Ising systems depends on the disorder distribution.

We study the critical behavior of the SRAM by means of Monte Carlo (MC) simulations. Since the model is essentially a spin glass we shall focus on the so-called overlap variables [15] $\sigma_{x} \tau_{x}$, where $\sigma_{x}$ and $\tau_{x}$ are associated with two different replicas of the model with the same bond variables. For the SRAM one can also consider the standard magnetic variables $\vec{s}_{x}=\sigma_{x} \vec{u}_{x}$. The MC results of [22] suggest that these quantities are not critical. We confirm here these conclusions so that little will be said about magnetic variables in the present study. We will study the behavior of the SRAM in the high-temperature phase. This reduces the algorithmic problems - the MC algorithm becomes very slow as temperature is reduced-and allows us to consider lattices of size $L^{3}$ up to $L=30$. In order to take into account finite-size effects we use the iterative method based on finite-size scaling (FSS) introduced in [25] and applied to disordered systems in [26, 27, 28, 29]. It allows us to obtain infinite-volume results up to $\xi_{\infty} \approx 20$ ( $\xi_{\infty}$ is the infinite-volume second-moment correlation length associated with the overlap two-point correlation function) in the high-temperature phase.

Our MC results do not show a direct evidence of a phase transition for $\beta \lesssim 1.00$, which is the range of values of $\beta$ that we can reliably simulate. On the other hand, our data clearly show FSS as $\beta$ increases, providing indirect evidence of the presence of a critical point. Fits of the infinite-volume results obtained for $\beta \lesssim 0.95$ indicate $\beta_{c}=1.08 \pm 0.04$. The corresponding critical exponents are:

$$
\eta_{o}=-0.24(4) \quad \nu_{o}=2.4(6) .
$$

They are defined by $\chi \sim \xi^{2-\eta_{o}}$ and $\xi \sim\left(\beta_{c}-\beta\right)^{-\nu_{o}}$; the suffix $o$ is introduced to remind that all exponents refer to the overlap variables and not to the magnetic ones. In the analysis it is crucial to include corrections to scaling in the FSS (corrections behave as $L^{-\omega}$ ) and in fits of infinite-volume quantities (nonanalytic corrections behave as $\xi_{\infty}^{-\omega}$ as $\left.\xi_{\infty} \rightarrow \infty\right)$. The exponent $\omega$ has been determined by studying the critical behavior of two universal ratios that involve the four-point and the two-point correlation function of the overlap variables. We obtain

$$
\omega=1.0 \pm 0.4 \text {. }
$$

Estimates (41) are reasonably close to those obtained for the ISGM (see Table 1 in [30] for a list of recent results) and thus support the conjecture that the SRAM transition is in the same universality class as that of the ISGM. Some additional arguments will be presented in Sec. 5. Similar conclusions were reached in [31] for the two-dimensional case. By using the large-cell RG method, it was shown that the two-dimensional SRAM has a zero-temperature transition with critical exponents compatible with those of the two-dimensional ISGM. 
The paper is organized as follows. In Sec. 2 we define the quantities that will be studied numerically. In Sec. 3 we discuss the MC algorithm. In Sec. 4 we discuss our MC simulations, providing evidence for the existence of a critical transition and computing the corresponding critical exponents. Finally, in Sec. 5 we draw our conclusions.

\section{Definitions}

We define here the quantities that have been determined in the $\mathrm{MC}$ simulation. The energy density and specific heat are defined as

$$
E \equiv \frac{1}{V} \overline{\langle-\mathcal{H}\rangle}, \quad C \equiv \frac{1}{V} \beta^{2}\left(\overline{\left\langle\mathcal{H}^{2}\right\rangle-\langle\mathcal{H}\rangle^{2}}\right)
$$

where $V \equiv L^{3}$ is the volume. In our numerical work we focus on the critical behavior of the overlap parameter

$$
q_{x} \equiv \sigma_{x} \tau_{x}
$$

where $\sigma_{x}$ and $\tau_{x}$ are two independent replicas of the system with the same couplings $j_{x y}$. We consider the correlation function $G(x) \equiv \overline{\left\langle q_{0} q_{x}\right\rangle}$, its Fourier transform $\widetilde{G}(p)$, the corresponding susceptibility $\chi$, and the second-moment correlation length $\xi$ :

$$
\chi \equiv \sum_{x} G(x)=\widetilde{G}(0), \quad \xi^{2} \equiv \frac{1}{4 \sin ^{2}\left(p_{\min } / 2\right)} \frac{\widetilde{G}(0)-\widetilde{G}(p)}{\widetilde{G}(p)},
$$

where $p=\left(p_{\min }, 0,0\right)$, and $p_{\min } \equiv 2 \pi / L$.

Moreover, we consider quartic correlations of the overlap parameter $q_{x}$ at zero momentum. Setting $\mu_{k} \equiv\left\langle\left(\sum_{x} q_{x}\right)^{k}\right\rangle$, we define the quartic susceptibilities

$$
V \chi_{4} \equiv \overline{\mu_{4}}-3 \overline{\mu_{2}^{2}}, \quad V \chi_{22} \equiv{\overline{\mu_{2}^{2}}}_{-\bar{\mu}_{2}^{2}} \text {, }
$$

and the quartic cumulant

$$
B_{q}=\frac{\overline{\mu_{4}}}{{\overline{\mu_{2}}}^{2}} .
$$

Finally, we define the zero-momentum four-point couplings

$$
G_{4} \equiv-\frac{\chi_{4}}{\xi^{3} \chi^{2}}, \quad G_{22} \equiv-\frac{\chi_{22}}{\xi^{3} \chi^{2}}
$$

We shall also briefly consider magnetic variables associated with $\vec{s}_{x}=\sigma_{x} \vec{u}_{x}$. In particular, if $\nu_{k} \equiv\left\langle\left(\sum_{x, y} \vec{s}_{x} \cdot \vec{s}_{y}\right)^{k / 2}\right\rangle$, we define the usual Binder cumulant

$$
B_{m} \equiv \frac{\overline{\nu_{4}}}{{\overline{\nu_{2}}}^{2}} \text {. }
$$

\section{The algorithm}

We consider model (3) on simple cubic lattices $L^{3}$ with periodic boundary conditions. No efficient algorithm is known for generic spin-glass systems (but recently progress has been made in some specific cases, see [32, 33, 34] and references therein), and thus we simply used the Metropolis algorithm with lexicographic choice of the lattice site. 
In order to reduce the thermalization times, which are the main source of bias in our simulations (and also the autocorrelation times, though this is not crucial, since the error on the results is mainly due to sample-to-sample fluctuations) we have combined it with the random-exchange method - also called parallel tempering or multiple Markov chain method -introduced in [35, 36, 37] (for a recent review with many different applications see 38; some improvements of the method are discussed in [39]).

In practice, we work as follows. We consider $N_{\beta}$ configurations at different inverse temperatures $\beta_{i}(\beta \equiv 1 / T), i=1, \ldots N_{\beta}$, in a given range $\left[\beta_{\min }, \beta_{\max }\right]$, that evolve according to Hamiltonian (3) with the same couplings $j_{x y}$. Every $N_{\text {ex }}$ iterations we perform $N_{\beta}-1$ random-exchange moves, trying to swap sequentially $\beta_{1}$ with $\beta_{2}$, then $\beta_{2}$ with $\beta_{3}$, up to $\beta_{N_{\beta}-1}$ and $\beta_{N_{\beta}}$. Each time we propose a swap of adjacent temperatures $\beta_{i}$ and $\beta_{i+1}$, which is accepted with probability $\operatorname{Min}\left\{1, \exp \left[\left(E_{i}-E_{i+1}\right)\left(\beta_{i}-\beta_{i+1}\right)\right]\right\}$, where $E_{i}$ is the energy of the configuration initially at inverse temperature $\beta_{i}$. For each sample we run $T_{\text {run }}$ Metropolis sweeps on each configuration and then repeat the same procedure for $N_{\text {sample }}$ different bond values. Note that, since we need to compute the overlap parameter, we simulated at the same time two different replicas of the system.

Thermalization represents the main source of bias in simulations of disordered systems. In all cases we start from a random infinite-temperature spin configuration. To check for equilibration, we used the following method. Let $\chi_{i}$ be the estimate of the overlap susceptibility $\chi_{i}$ at iteration $i$ at the largest $\beta$ value, $\beta_{\max }$, averaged over the $N_{\text {sample }}$ disorder realizations. Then, define a block length $T_{\text {block }}$ and the block-averaged quantities

$$
\chi_{b, i}=\frac{1}{T_{\text {block }}} \sum_{j=1}^{T_{\text {block }}} \chi_{j+(i-1) T_{\text {block }}}
$$

In our runs we typically choose $T_{\text {block }}=5000$ or 10000 (all results presented below are always in units of Metropolis sweeps). In the tests we report below, in order to determine the thermalization times more precisely, we use $T_{\text {block }}=2000$. Finally, plot $\chi_{b, i}$ as a function of $i$. For $i$ large, $\chi_{b, i}$ becomes constant within error bars, signalling equilibration. Data outside the approximately flat region are discarded in the calculation of the mean values.

In the algorithm there are several parameters that must be tuned: $\beta_{\min }, \beta_{\max }$, $N_{\beta}$, and $N_{\text {ex }}$. Moreover, $T_{\text {run }}$ should be large enough to reach equilibration. In our simulations the difference $\Delta \beta$ between adjacent $\beta$ values is kept constant, so that $\beta_{\max }-\beta_{\min }=\left(N_{\beta}-1\right) \Delta \beta$. The highest-temperature value $\beta_{\min }$ must be chosen such that, at this value of $\beta$, the standard Metropolis algorithm is reasonably efficient. In most of our simulations we use $\beta_{\min }=0.81$. At this value of $\beta$ (it corresponds to an infinitevolume correlation length $\xi_{\infty} \approx 3.7$ ) the Metropolis dynamics is reasonably fast. Then, at fixed $\beta_{\min }$ and $\beta_{\max }$, we investigated how the thermalization time depends on the two parameters $N_{\beta}$ (or, equivalently, $\Delta \beta$, since we work in a fixed $\beta$ interval) and $N_{\text {ex }}$. The parameter $\Delta \beta$ controls the acceptance rate of the random-exchange moves and should not be too large, otherwise there are no temperature swaps and the exchange dynamics 
becomes slow. As we shall see, $\Delta \beta$ is not a parameter that requires detailed tuning, since there is a somewhat large interval of $\Delta \beta$ values for which the algorithm is equally efficient, i.e. that all correspond to the minimal thermalization time (even though the acceptance rates change significantly). In order to perform a quantitative comparison, we define the thermalization time $T_{\mathrm{th}, x \%}$ as the time such that $\left|\chi_{b, i} / \chi_{\text {ave }}-1\right|=x / 100$, where $i=T_{\mathrm{th}, x \%} / T_{\text {block }}$ and $\chi_{\text {ave }}$ is the average value of $\chi$ at $\beta_{\max }$. Since the typical errors on $\chi$ are $\approx 1 \%$ we consider $x \%=2 \%$. The thermalization time $T_{\mathrm{th}, x \%}$ is of course a lower bound on the true thermalization time, but it has the advantage of being computationally well-defined and thus it allows quantitative comparisons of the results corresponding to different choices of the parameters. Moreover, we define a first roundtrip time $T_{\text {frt }}$ (in units of Metropolis sweeps): it corresponds to the time needed by a given configuration to go at least once through all temperatures. For each simulation we collected $2 N_{\beta} N_{\text {sample }}$ first round-trip times. Their distribution is not Gaussian, but it has an exponential tail $e^{-c y}$. Therefore, instead of the average, we found more informative to consider the time $T_{\text {frt, } 90 \%}$ such that $90 \%$ of the measured $2 N_{\beta} N_{\text {sample }}$ first roundtrip times is smaller than $T_{\text {frt, } 90 \%}$. In our tests we found $T_{\text {frt, } 90 \%}$ to be related to the thermalization time. Equilibration is reached after a few $T_{\mathrm{frt}, 90 \%}$.

In Fig. 1, for several values of $\Delta \beta$, we show some data corresponding to simulations for $L=16, N_{\text {sample }}=1000, \beta_{\min }=0.81, \beta_{\max }=1.09$, and $N_{\mathrm{ex}}=10$. At the top we show the acceptance range. Since $\Delta \beta$ is kept fixed, the acceptance rate is not constant. The minimum (maximum) value corresponds to swaps of the configurations with the smallest (largest) $\beta$. The acceptance rate for the intermediate $\beta$ values varies approximately linearly. In the figure we also show the first round-trip time $T_{\text {frt,90\% }}$, the thermalization time $T_{\mathrm{th}, 2 \%}$ (in all cases we used $\chi_{\text {ave }}=644(3)$ as estimate of $\chi$ at $\left.\beta_{\max }=1.09\right), T_{\text {comp }} \approx N_{\beta} T_{\mathrm{th}, 2 \%}$, which is roughly proportional to the computer time. The uncertainty on these quantities should be approximately $10 \%$. The data show a significant correlation between the thermalization and round-trip times. Their ratio is approximately 1.5-2, indicating that a few round trips are needed (and sufficient) to thermalize the system. The thermalization time has a minimum in a relatively large region of $\Delta \beta$ values, i.e., for $0.02 \lesssim \Delta \beta \lesssim 0.05$, corresponding to average acceptance rates $10-50 \%$. This suggests that the thermalization time $T_{\text {th }}$ does not depend much on the acceptance rate as long as it is not too small (say, larger than 10\%). If we consider the computer time - and this is what we are really interested in - the optimal region is shifted to larger values of $\Delta \beta$. The minimum corresponds to approximately $\Delta \beta=0.04$, where the acceptance rate varies in the interval [0.115, 0.312]. However, an additional increase in $\Delta \beta$ does not worsen much the efficiency of the algorithm that is nearly optimal even for $\Delta \beta=0.07$, where the acceptance rate is rather small (it varies between $0.7 \%$ and $7 \%$ ).

The second interesting parameter is $N_{\text {ex }}$. We compare here two simulations with $L=16, N_{\text {sample }}=1000$ using $N_{\text {ex }}=10,20$ and keeping fixed the other parameters at $\beta_{\min }=0.81, \beta_{\max }=1.09$, and $\Delta \beta=0.04$. We find $T_{\mathrm{th}, 2 \%} \approx 16000$ and $T_{\mathrm{frt}, 90 \%} \approx 9000$ for $N_{\mathrm{ex}}=10$, and $T_{\mathrm{th}, 2 \%} \approx 28000$ and $T_{\mathrm{frt}, 90 \%} \approx 18000$ for $N_{\mathrm{ex}}=20$. Clearly, $N_{\mathrm{ex}}=10$ 


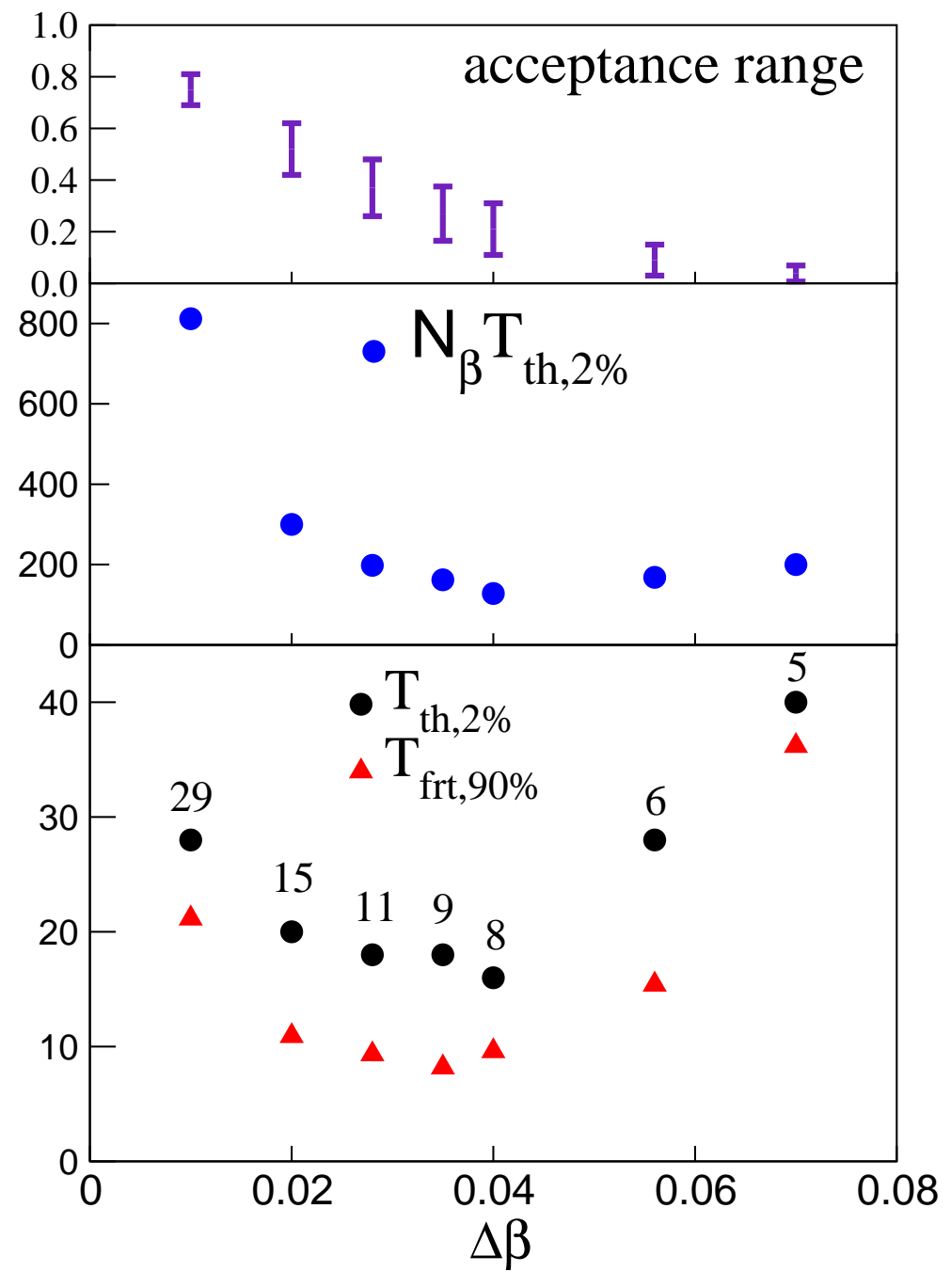

Figure 1. We show the acceptance range (above), the quantity $N_{\beta} T_{\text {th, } 2 \%}$ (in units of 1000 sweeps) which is roughly proportional to the computer time (middle), the thermalization time $T_{\mathrm{th}, 2 \%}$ and the first round-trip time $T_{\text {frt }, 90 \%}$, both in units of 1000 sweeps, (below) versus $\Delta \beta$ for random-exchange runs with $L=16, \beta_{\min }=0.81$, $\beta_{\max }=1.09, N_{\mathrm{ex}}=10$. The numbers reported close to the estimates of $T_{\mathrm{th}, 2 \%}$ correspond to the number $N_{\beta}$ of $\beta$ values.

is better than $N_{\mathrm{ex}}=20$. This indicates that $N_{\mathrm{ex}}$ should be taken relatively small. In our simulations we fixed $N_{\text {ex }}=10$.

As expected, the thermalization time depends very strongly on $\beta_{\max }$. For example, for $L=16, \beta_{\min }=0.81$, and $\Delta \beta=0.01$, we find $T_{\mathrm{th}, 2 \%} \approx 16000$ for $\beta_{\max }=1.00$ and $T_{\mathrm{th}, 2 \%} \approx 28000$ for $\beta_{\max }=1.09$. Note that that the acceptance rates are similar varying from 0.686 (for $\beta=0.81,0.82$ ), to 0.772 (for $\beta=0.99,1.00$ ) and 0.805 (for $\beta=1.08,1.09$ ). We have performed an analogous test for $L=24$. For $\beta_{\min }=0.81$, $\Delta \beta=0.01, N_{\mathrm{ex}}=10$, we find $T_{\mathrm{frt}, 90 \%} \approx 18000$ and $T_{\mathrm{th}, 2 \%} \lesssim 70000$ for $\beta_{\max }=1.0$, $T_{\mathrm{frt}, 90 \%} \approx 40000$ and $T_{\mathrm{th}, 2 \%}>100000$ for $\beta_{\max }=1.05$.

Finally, it is interesting to compare the thermalization times for a Metropolis 
Table 1. Our runs using the random-exchange method. Here $T_{\text {run }}$ is the number of Metropolis sweeps per sample and $T_{\text {disc }}$ is the number of discarded Metropolis sweeps. In all cases $N_{\mathrm{ex}}=10$.

\begin{tabular}{ccccrc}
\hline$L$ & $\beta_{\min }, \beta_{\max }$ & $\Delta \beta$ & $N_{\text {sample }}$ & \multicolumn{1}{c}{$T_{\text {run }}$} & $T_{\text {disc }}$ \\
\hline 12 & $0.90,1.10$ & 0.02 & 5000 & 50000 & 40000 \\
12 & $0.91,1.11$ & 0.02 & 5000 & 50000 & 40000 \\
16 & $0.81,1.00$ & 0.01 & 5000 & 50000 & 40000 \\
18 & $0.80,1.04$ & 0.02 & 2000 & 100000 & 40000 \\
18 & $0.81,1.05$ & 0.02 & 2000 & 100000 & 60000 \\
20 & $0.76,0.80$ & 0.01 & 2000 & 50000 & 10000 \\
20 & $0.81,0.95$ & 0.01 & 2000 & 100000 & 40000 \\
24 & $0.81,1.00$ & 0.01 & 2000 & 100000 & 80000 \\
30 & $0.76,0.80$ & 0.01 & 2000 & 50000 & 16000 \\
30 & $0.81,0.95$ & 0.01 & 1500 & 100000 & 90000 \\
30 & $0.81,0.95$ & 0.01 & 500 & 150000 & 90000 \\
\hline
\end{tabular}

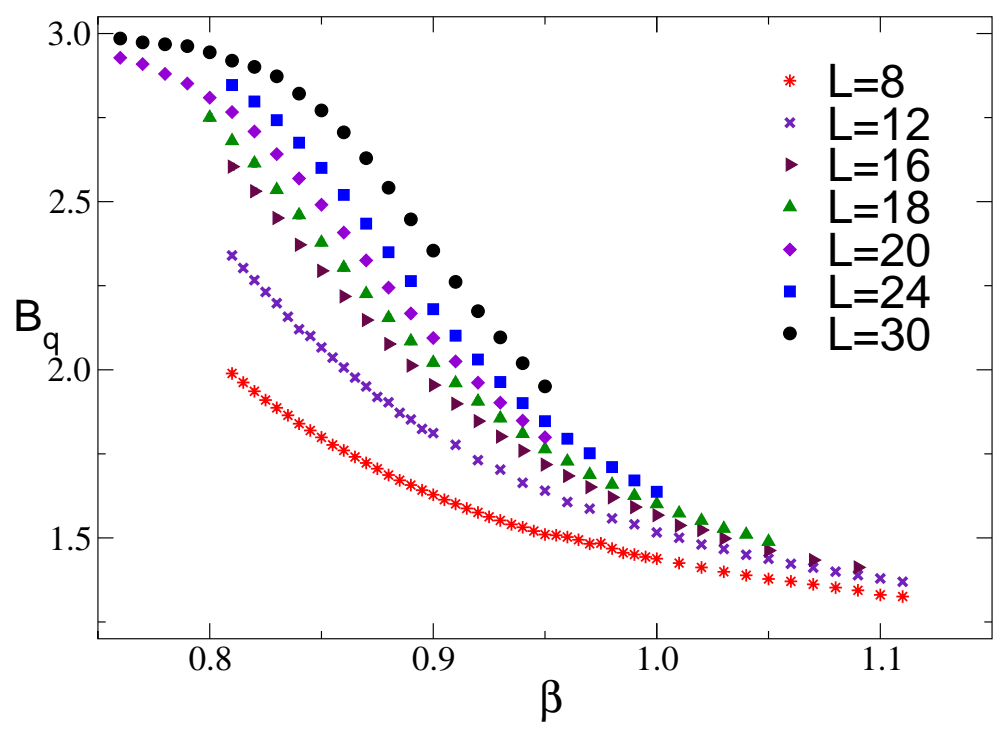

Figure 2. The quartic cumulant $B_{q}$ of the overlap parameter for several lattice sizes.

simulation without random-exchange moves and a random-exchange simulation. For $L=16$ and $\beta=1.0$ we performed a long Metropolis simulation, considering 5000 disorder realizations. The thermalization time $T_{\mathrm{th}, 2 \%}$ is approximately $400 \cdot 10^{3}$. This should be compared with a random-exchange run with $\beta \in[0.81,1.09], \Delta \beta=0.04$. In this case $T_{\mathrm{th}, 2 \%} \approx 16 \cdot 10^{3}$. Even if the random-exchange run has a larger $\beta_{\max }$, the thermalization time is much smaller. 


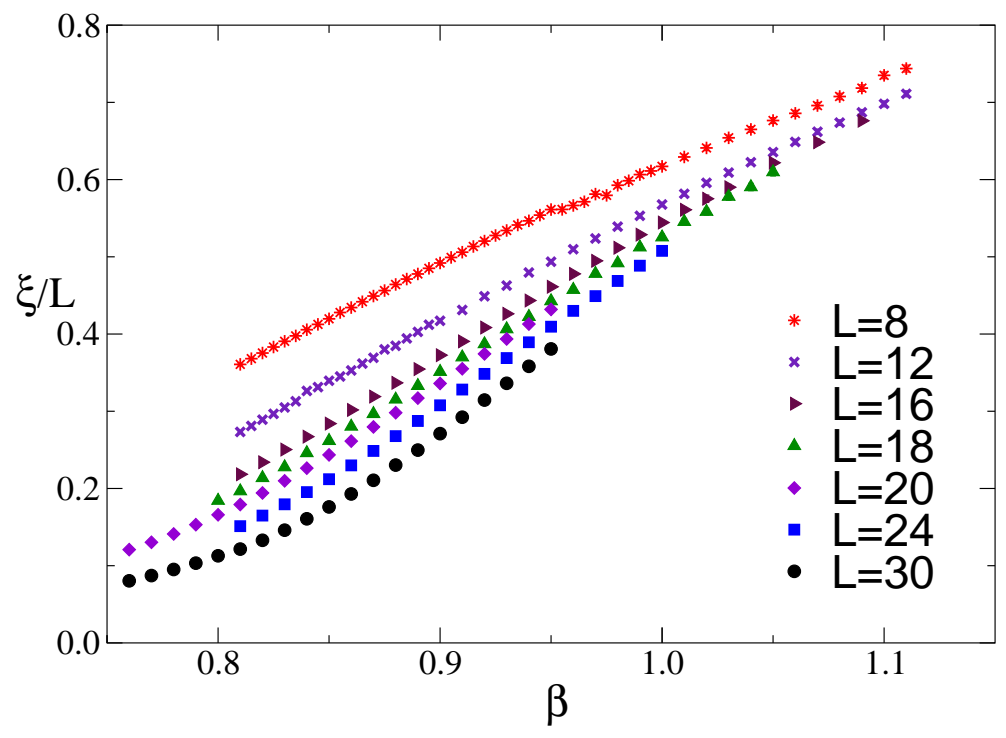

Figure 3. The ratio $\xi / L$ for several lattice sizes.

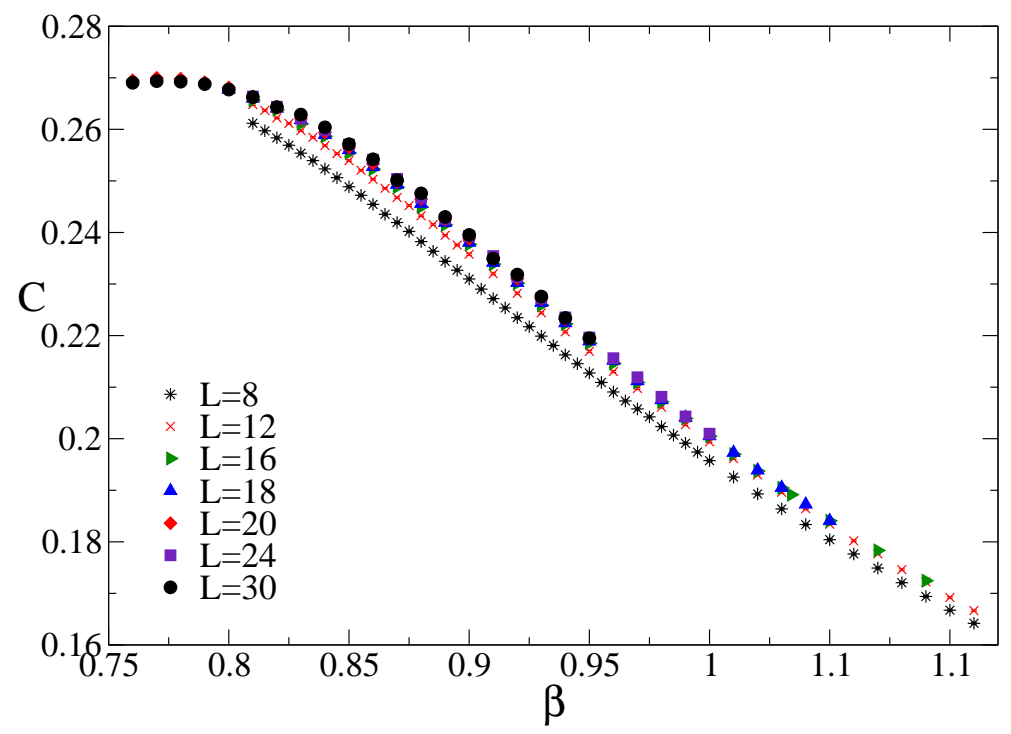

Figure 4. The specific heat for several lattice sizes.

\section{Results}

We consider model (3) on simple cubic lattices $L^{3}$ with periodic boundary conditions. We have performed a series of simulations for lattices with $8 \leq L \leq 30$, using the random-exchange method, as explained in Sec. 3. For each value of $L$ we considered a range of $\beta$ values such that the thermalization time was less than $10^{5}$ Metropolis sweeps per sample. We could therefore take $T_{\text {run }} \approx 10^{5}$. We required this condition in order to be able to have $N_{\text {sample }} \geq 1000$, and thus precise estimates. Of course, this limits the parameter $\beta_{\max }$ and, for $L=30$, we were able to collect data only up to $\beta=0.95$. The parameters of our random-exchange runs are reported in Table 1] We are not reporting 


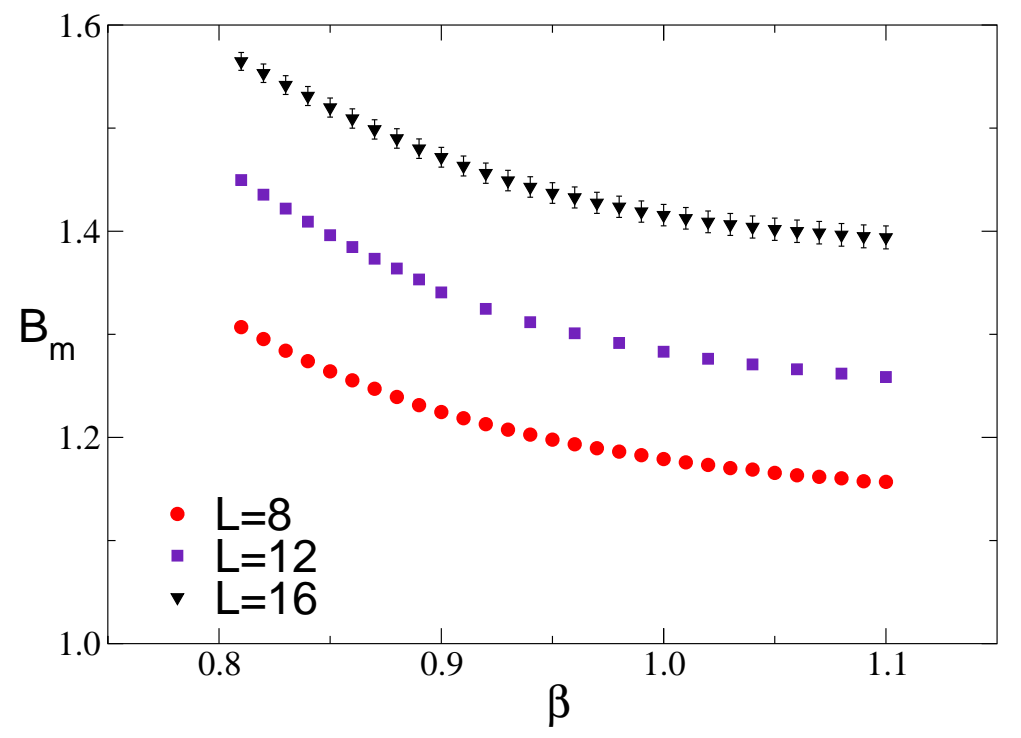

Figure 5. The magnetic quartic cumulant $B_{m}$ for several lattice sizes.

several other runs with $L=16, N_{\text {sample }}=1000$, that have been used in the numerical tests reported in Sec. 3. Few runs with $L=8,12,16$ were performed by using only Metropolis updatings without random-exchange moves. In Table 1 we report the length $T_{\text {run }}$ of the run for each value of the parameters and $T_{\text {disc }}$, the number of sweeps that have been discarded before measuring (this parameter has been chosen conservatively to avoid any thermalization bias). Simulations took approximately 2.5 CPU years of a workstation equipped with an AMD Opteron Processor 246 (2 GHz clock).

In Figs. 2 and 3 we show the quartic cumulant $B_{q}$ defined in Eq. (10) and the ratio $\xi / L$ for several lattice sizes $L$. In the region of $L$ and $\beta$ covered by our MC data, neither $B_{q}$ nor $\xi / L$ have a crossing point. Thus, we do not have direct evidence for a finite-temperature transition in the range of temperatures that we can reliably simulate. No indication of a phase transition in this range of $\beta$ is also provided by the results for the specific heat - they are shown in Fig. 4-and by magnetic variables - there is no indication of a crossing point in the results for $B_{m}$ defined in Eq. (12), see Fig. 5 ,

These results are consistent with two possible scenarios: (i) the system becomes critical at $\beta=\beta_{c}$ with $\beta_{c} \gtrsim 1.05$ (with the possibility of a zero-temperature transition, i.e. $\left.\beta_{c}=+\infty\right)$; (ii) the system never shows criticality and even for $\beta=\infty$ the correlation length is finite. We will now show that our data allow us to exclude this second possibility since, as $\beta$ increases, the model shows critical behavior; more precisely, our data for the overlap variables show FSS as expected close to a critical point. In order to make the check as reliable as possible we will study the FSS behavior of the ratios $A(\beta, s L) / A(\beta, L)$, where $A(\beta, L)$ is a long-distance quantity and $s$ is an arbitrary number. In the FSS limit (i.e. for $L, \xi(\beta, L) \rightarrow \infty$ at $\xi(\beta, L) / L$ fixed) we should have

$$
\frac{A(\beta, s L)}{A(\beta, L)} \longrightarrow F_{A}[s, \xi(\beta, L) / L],
$$




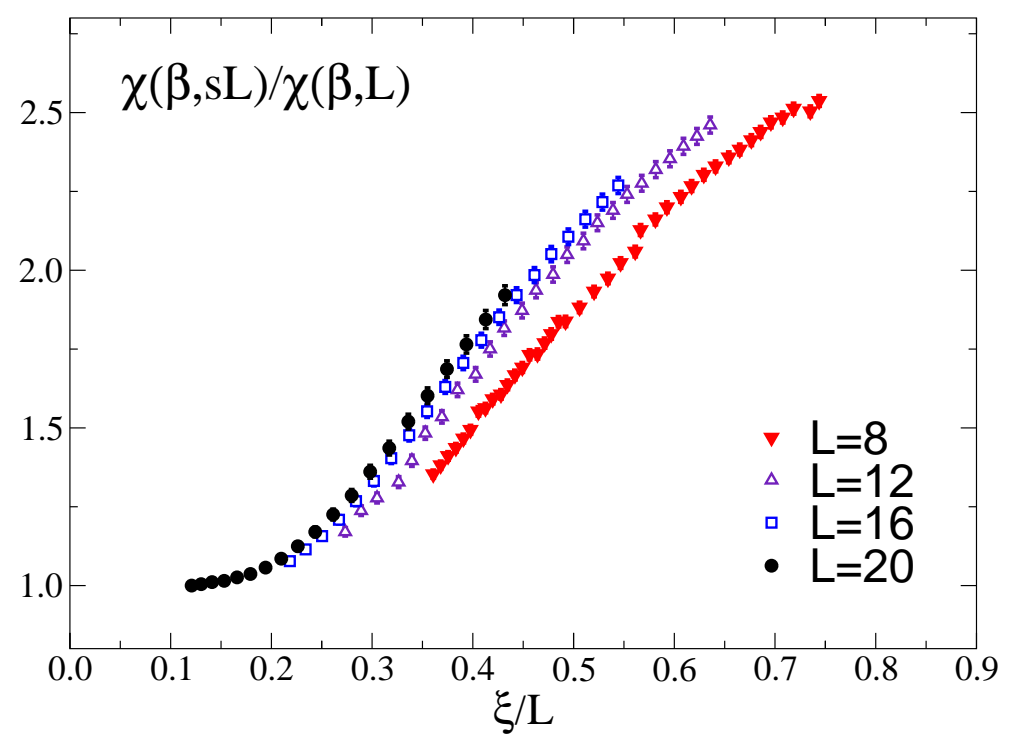

Figure 6. The FSS curve of the susceptibility $\chi$ for $s=3 / 2$.

where $F_{A}(s, z)$ is a universal function. Note that in this formulation there are no free parameters and thus one can make an unbiased test of FSS.

The FSS curves computed by using Eq. (14) can be used to determine infinitevolume quantities for large values of the correlation length. For this purpose we employ the extrapolation method of [25], (see also [40, 41] for a discussion of the efficiency of this technique). Indeed, in the absence of scaling corrections, Eq. (14) allows us to compute $A(\beta, s L)$ on a lattice of size $s L$ in terms of quantities defined on a lattice of size $L$ and of the function $F_{A}(s, z)$. In practice, one works as follows. First, one performs several runs, determining $A(\beta, s L), A(\beta, L), \xi(\beta, s L)$, and $\xi(\beta, L)$. By means of a suitable interpolation, this provides the FSS function $F_{A}(s, z)$ for $A$ and $\xi$. Then, $A_{\infty}(\beta)$ and $\xi_{\infty}(\beta)$ are obtained from $A(\beta, L)$ and $\xi(\beta, L)$ by iterating Eq. (14) and the corresponding equation for $\xi(\beta, L)$. Of course, one must be very careful about scaling corrections, discarding systematically lattices with small values of $L$ till results become independent of $L$ within error bars.

In Figs. 6, 7] 8, and 9 we plot the ratios (14) for $\chi, \xi, G_{4}$, and $G_{22}$, fixing $s=3 / 2$. The curves for $\chi$ and $\xi$ show significant scaling corrections. However, they apparently decrease very rapidly with $L$ and indeed the data corresponding to the pairs $L=16,24$ are only slightly different from those with $L=20,30$. Therefore, they strongly suggest that FSS holds, although, for $L \lesssim 30$, scaling corrections are significant compared to our error bars (for $\chi$ our data have a relative error of approximately $1 \%$ for $L=20$ and $L=30)$. For $G_{4}$ and $G_{22}$ corrections are apparently weaker and indeed the data corresponding to $L=20,30$ are compatible with those with $L=12,18$ and with $L=16,24$. In this case FSS holds within error bars.

The presence of scaling corrections in the FSS curve for $\xi$ does not allow us to use straightforwardly the iteration method of [25] and makes it necessary to include scaling 


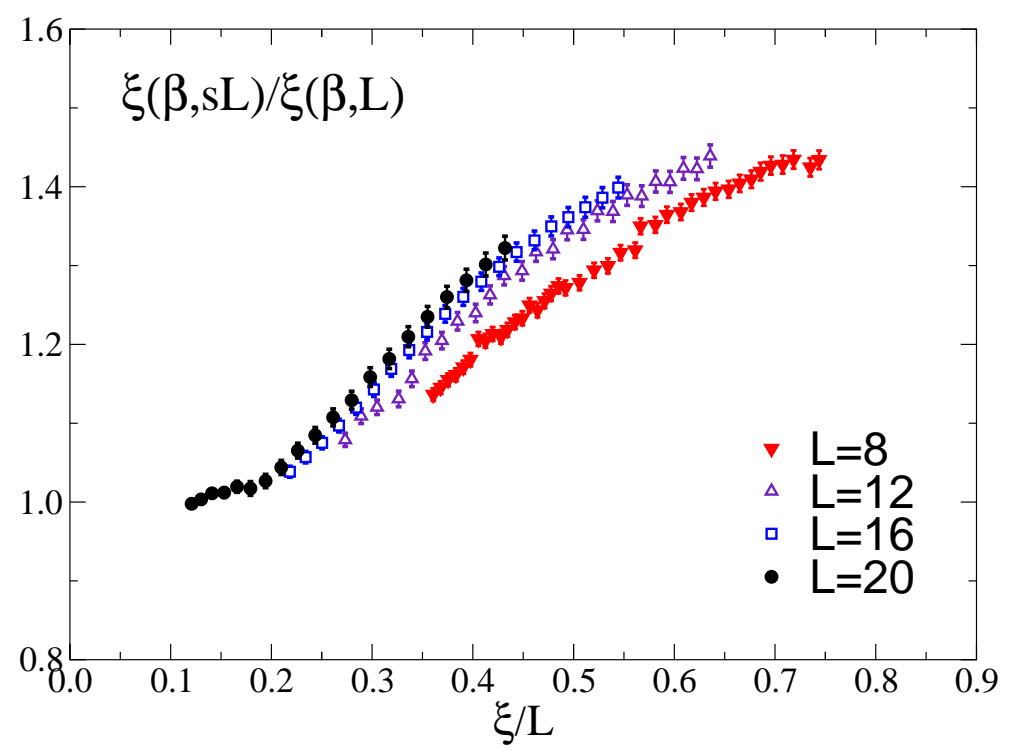

Figure 7. The FSS curve of the correlation length $\xi$ for $s=3 / 2$.

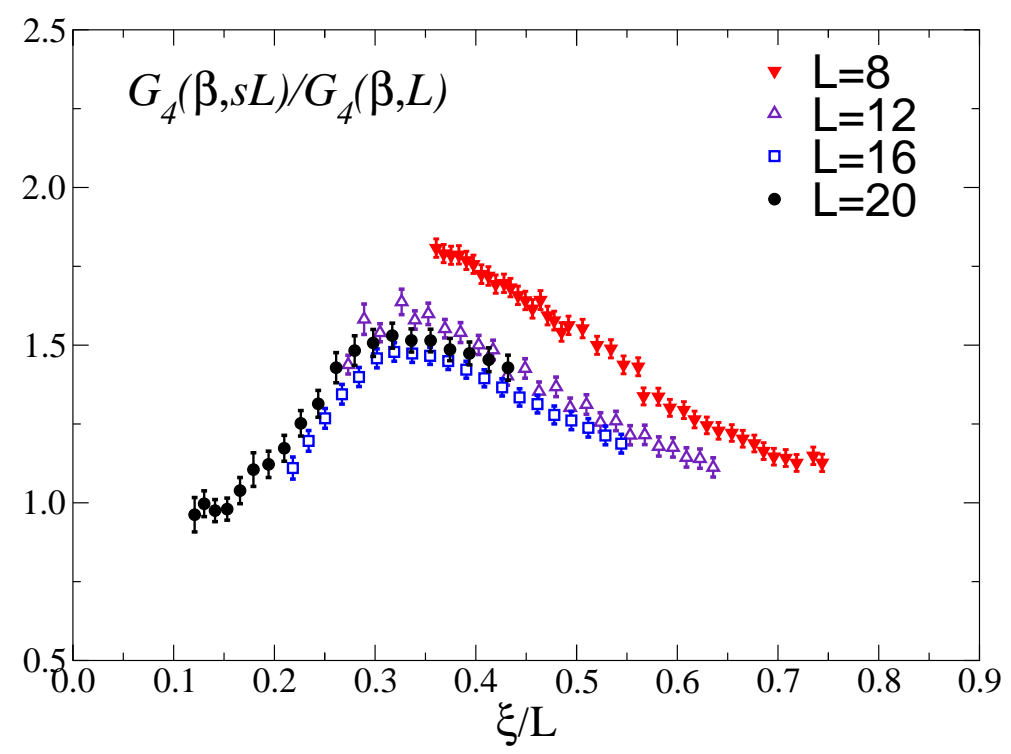

Figure 8. The FSS curve of the zero-momentum quartic coupling $G_{4}$ for $s=3 / 2$.

corrections in the scaling Ansatz. As done in [27], we use a more general Ansatz of the form

$$
\frac{A(\beta, s L)}{A(\beta, L)}=F_{A}(s, \xi(\beta, L) / L)+L^{-\omega} G_{A}(s, \xi(\beta, L) / L),
$$

where $G_{A}(s, z)$ is a new universal scaling function and $\omega$ is a to-be-determined correctionto-scaling exponent.

Our data are not precise enough to allow a determination of $\omega$. However, it is easy to convince oneself that $\omega$ cannot be arbitrarily large. If we consider the correlation length $\xi(\beta, L)$, the correction-to-scaling exponent should be less than 2 , since corrections 


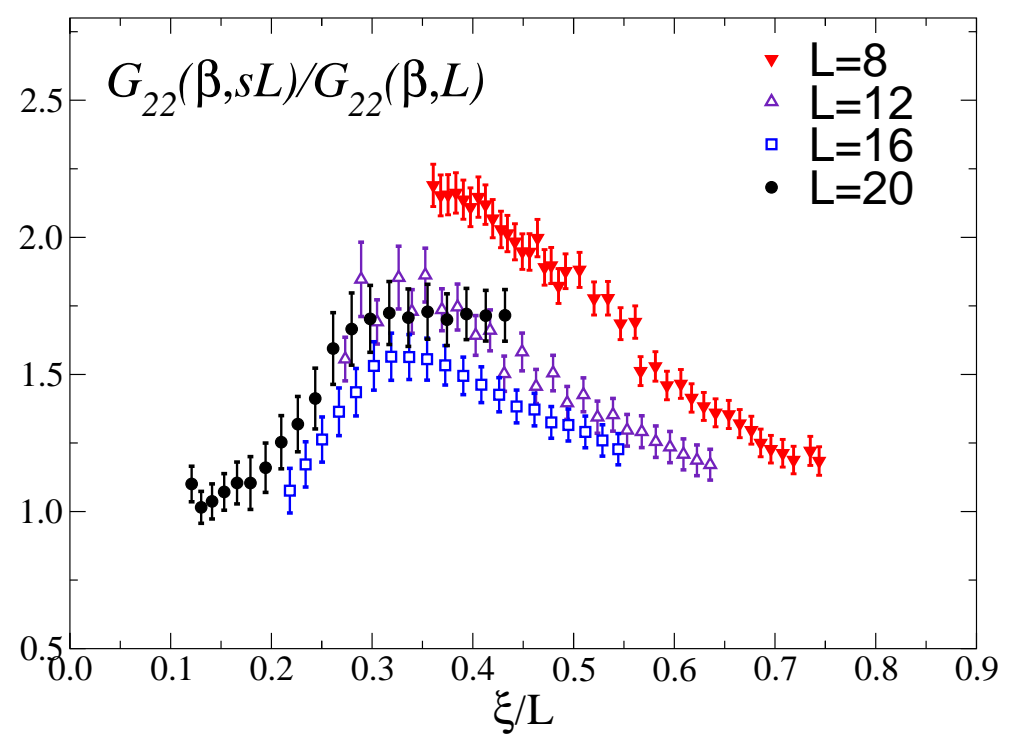

Figure 9. The FSS curve of the zero-momentum quartic coupling $G_{22}$ for $s=3 / 2$.

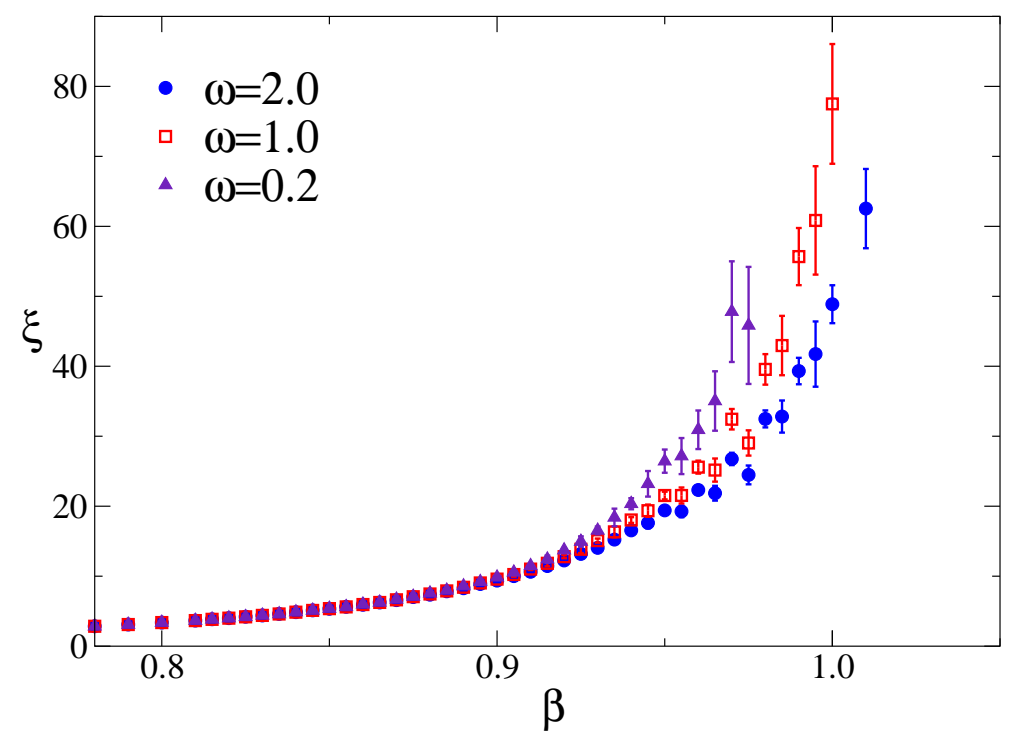

Figure 10. The infinite-volume correlation length $\xi_{\infty}$ as obtained by the extrapolation FSS method.

with exponent 2 appear necessarily [42. They are related to the very definition of the second-moment correlation length we use on a finite lattice. Indeed, it corresponds to its infinite-volume counterpart only up to terms of order $L^{-2}$. For $\chi, G_{4}$, and $G_{22}$ there are corrections due to the analytic background [43, 44] that are proportional to $L^{2-\eta_{o}}$, where $\eta_{o}$ is the susceptibility exponent, $\chi \sim \xi^{2-\eta_{o}}$. As we shall see, $\eta_{o} \approx-0.3$, so that $\omega \lesssim 2.3$. On the other hand, one cannot set a priori a lower bound on $\omega$ and, in principle, $\omega$ can be arbitrarily small. We will present below analyses with $\omega$ as small as 0.2 , presenting results for $\omega=0.2, \omega=1$, and $\omega=2$. This choice will be justified below. 


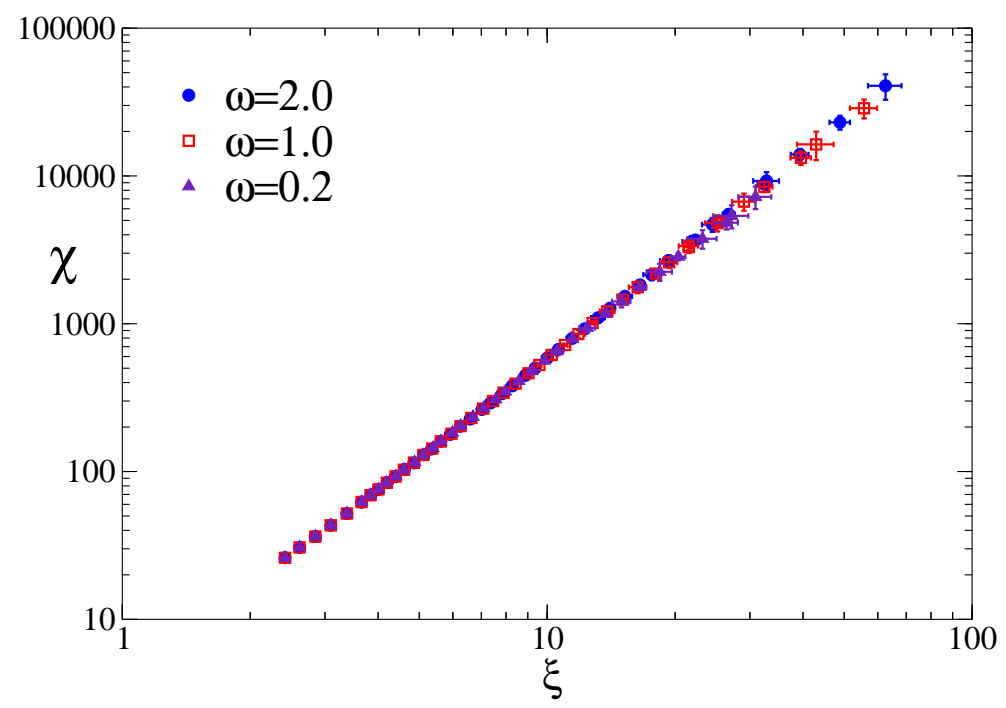

Figure 11. Log-log plot of the infinite-volume estimates of $\chi_{\infty}$ versus $\xi_{\infty}$.

In order to determine $\eta_{o}$, we consider the data reported in Figs. 6] and 7 and apply the extrapolation technique using Eq. (15). As in 25] the scaling curves are parametrized as polynomials in $\exp [-\xi(\beta, L) / L]$ satisfying $G_{A}(s, 0)=F_{A}(s, 0)=1$. A fourth-order polynomial for $F_{A}(s, z)$ and a third-order polynomial for $G_{A}(s, z)$ appear to be adequate. In Fig. 10 we show the infinite-volume correlation length $\xi_{\infty}(\beta)$. It can be determined reliably up to $\beta=0.95$, essentially because we have results for the largest lattice, $L=30$, only up to this value of $\beta$. The dependence on $\omega$ is not large, but in any case larger that the statistical errors. For $\beta=0.95$, we find $\xi_{\infty}=26.4(1.7), 21.5(5)$, $19.4(4)$ for $\omega=0.2,1,2$, respectively. Whatever is the value of $\omega, \xi_{\infty}(\beta)$ increases quite rapidly, confirming that the system eventually becomes critical. Evidence of criticality is also provided in Fig. 11] where we show a log-log plot of $\chi_{\infty}$ versus $\xi_{\infty}$. The behavior is linear, indicating that

$$
\chi_{\infty} \sim \xi_{\infty}^{2-\eta_{o}},
$$

where $\eta_{o}$ is a critical exponent. Note that the dependence on $\omega$ of $\chi_{\infty}$ at fixed $\xi_{\infty}$ is much smaller than the $\omega$ dependence of $\chi_{\infty}$ and $\xi_{\infty}$ at fixed $\beta$. This is due to the fact that, at fixed $\beta, \chi_{\infty}$ and $\xi_{\infty}$ are strongly correlated: they both increase with decreasing $\omega$, in such a way that the ratio $\chi_{\infty} / \xi_{\infty}^{2-\eta_{o}}$ has a tiny dependence on $\omega$. To obtain an estimate of $\eta_{o}$ we fitted $\chi_{\infty}$ and $\xi_{\infty}$ to $\ln \chi_{\infty}=a+\left(2-\eta_{o}\right) \ln \xi_{\infty}$. To estimate the scaling corrections, the fit has been repeated several times, each time including only the data satisfying $\beta \geq \beta_{\min }$. Results are reported in Table 2 for different values of $\omega$. In order to have an additional check on the stability of the results, we have also repeated the analysis twice: we present results obtained by using all data $\left(L_{\min }=8\right)$ and results obtained using only lattices with $L \geq 12\left(L_{\min }=12\right)$.

The results presented in Table 2 depend somewhat on $\omega$ and $\beta_{\min }$. At fixed $\omega, \eta_{o}$ decreases with increasing $\beta_{\min }$, reaching an approximate plateau within error bars for $\beta_{\min } \gtrsim 0.84$. At fixed $\beta_{\min }$ the estimates decrease with increasing $\omega$. A conservative 
Table 2. Estimates of the exponent $\eta_{o}$ for several values of $\omega$ and $L_{\min }$.

\begin{tabular}{lllllll}
\hline & \multicolumn{3}{c}{$L_{\min }=8$} & & & $L_{\min }=12$ \\
$\beta_{\min }$ & $\omega=0.2$ & $\omega=1$ & $\omega=2$ & $\omega=0.2$ & $\omega=1$ & $\omega=2$ \\
\hline 0.76 & $-0.161(7)$ & $-0.177(6)$ & $-0.185(6)$ & $-0.148(9)$ & $-0.159(8)$ & $-0.167(7)$ \\
0.78 & $-0.191(9)$ & $-0.203(8)$ & $-0.214(7)$ & $-0.175(11)$ & $-0.185(9)$ & $-0.196(9)$ \\
0.80 & $-0.210(12)$ & $-0.221(10)$ & $-0.233(9)$ & $-0.196(15)$ & $-0.203(12)$ & $-0.216(11)$ \\
0.82 & $-0.221(15)$ & $-0.234(12)$ & $-0.247(10)$ & $-0.205(21)$ & $-0.214(15)$ & $-0.227(14)$ \\
0.84 & $-0.236(22)$ & $-0.251(16)$ & $-0.268(14)$ & $-0.225(31)$ & $-0.234(22)$ & $-0.242(19)$ \\
0.86 & $-0.233(32)$ & $-0.258(22)$ & $-0.276(19)$ & $-0.217(50)$ & $-0.233(31)$ & $-0.251(27)$ \\
0.88 & $-0.236(50)$ & $-0.265(31)$ & $-0.288(26)$ & $-0.213(81)$ & $-0.241(45)$ & $-0.262(38)$ \\
\hline
\end{tabular}

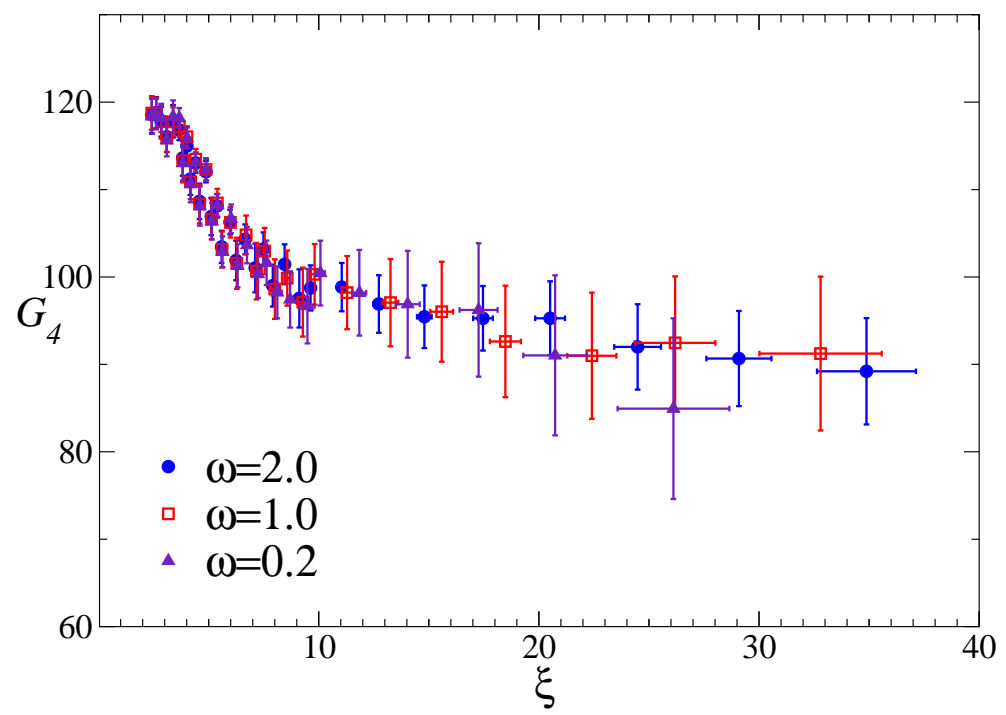

Figure 12. Estimates of $G_{4, \infty}$ versus $\xi_{\infty}$ for three different values of $\omega$.

estimate can be obtained by noting that all results with $0.84 \lesssim \beta_{\text {min }} \lesssim 0.86$ - for larger values of $\beta_{\text {min }}$ error bars are quite large - lie in the interval $-0.28 \lesssim \eta_{o} \lesssim-0.18$, including statistical errors. We thus end up with the following estimate:

$$
\eta_{o}=-0.23(5) \text {. }
$$

Then, we consider the RG-invariant quantities $G_{4}$ and $G_{22}$. Again, we use Eq. (15) and the iteration algorithm to determine infinite-volume estimates $G_{4, \infty}(\beta)$ and $G_{22, \infty}(\beta)$. Reasonable results are only obtained if the data with $L=8$ are discarded, i.e. if we only consider $L \geq L_{\min }=12$. Indeed, if all MC data are used in the FSS extrapolation, $G_{4, \infty}(\beta)$ and $G_{22, \infty}(\beta)$ apparently do not converge to a finite value as $\beta \rightarrow \infty$. For $L_{\min }=12$, the $\omega$ dependence is of the order of the statistical error bars: for instance, for $\beta=0.95$ we predict $G_{4, \infty}=85(10), 91(7), 95(4)$, and $G_{22, \infty}=-13.4(7),-13.1(7)$, $-11(2)$, for $\omega=0.2,1,2$. The results are shown in Figs. 12 and 13, They show a 


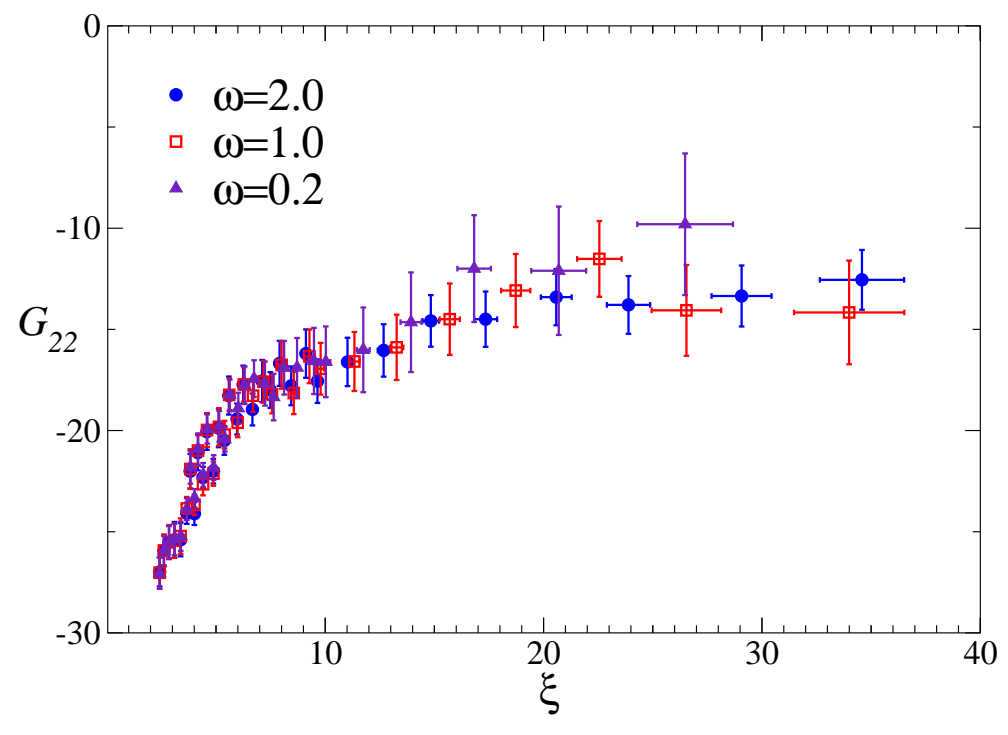

Figure 13. Estimates of $G_{22, \infty}$ versus $\xi_{\infty}$ for three different values of $\omega$.

rapid variation with $\xi_{\infty}$ for small values of the correlation length and reach a plateau for $\xi_{\infty} \gtrsim 15$. In order to estimate the critical value for $\xi_{\infty} \rightarrow \infty$, we perform a fit of the form

$$
\mathcal{R}_{\infty}=\mathcal{R}^{*}+\frac{a_{\mathcal{R}}}{\xi_{\infty}^{\Omega}}
$$

where $\mathcal{R}^{*}, a_{\mathcal{R}}$, and $\Omega$ are free parameters, $\mathcal{R}=G_{4}$ or $G_{22}$. Results are reported in Tables 3 and 4. The dependence on $\omega$ is here quite small, as it has to be expected since there is no evidence of scaling corrections in the FSS curves for $G_{4}$ and $G_{22}$. The dependence we observe here is mainly related to the corrections affecting the correlation length that is also used in the extrapolation. Again our final estimate is obtained conservatively by looking at the variation with $\omega$ and $\beta_{\min }$. For $G_{4}^{*}$, we take as our final estimate

$$
G_{4}^{*}=88(8),
$$

where the error is such to include all results with $\beta_{\min } \geq 0.81$. Analogously, we estimate

$$
G_{22}^{*}=-11(4)
$$

The fit parameter $\Omega$ that appears in Eq. (18) is not completely arbitrary and can be related to the exponent $\omega$ used in the FSS extrapolation. Thus, the results of Tables 3 and 4 can be used to obtain constraints on the value of $\omega$. In general, given an infinitevolume RG-invariant quantity $\mathcal{R}(\beta)$, close to a critical point we expect an expansion of the form:

$$
\mathcal{R}(\beta)=\mathcal{R}^{*}\left(1+a\left|\beta-\beta_{c}\right|^{\Delta}\right),
$$

with a positive correction-to-scaling exponent $\Delta$. According to the RG theory, scaling corrections may have several origins [45, 46]:

(i) There are analytic corrections of the form $\left|\beta-\beta_{c}\right|^{n}, n$ being an integer. 
Table 3. Estimates of $G_{4}^{*}$ and $\Omega$ for different values of $\omega$. Here $L_{\min }=12$.

\begin{tabular}{clllccc}
\hline \multicolumn{3}{c}{$\omega=0.2$} & \multicolumn{2}{c}{$\omega=1$} & \multicolumn{2}{c}{$\omega=2$} \\
$\beta_{\min }$ & \multicolumn{1}{c}{$G_{4}^{*}$} & \multicolumn{1}{c}{$\Omega$} & $G_{4}^{*}$ & $\Omega$ & $G_{4}^{*}$ & $\Omega$ \\
\hline 0.780 & $58(26)$ & $0.36(19)$ & $72(13)$ & $0.47(17)$ & $78(8)$ & $0.55(15)$ \\
0.790 & $74(13)$ & $0.58(23)$ & $78(9)$ & $0.58(19)$ & $83(6)$ & $0.70(18)$ \\
0.800 & $85(7)$ & $0.95(30)$ & $86(6)$ & $0.89(25)$ & $87(4)$ & $0.93(21)$ \\
0.810 & $87(6)$ & $1.08(34)$ & $87(5)$ & $1.01(29)$ & $88(4)$ & $0.98(23)$ \\
0.815 & $86(7)$ & $1.00(38)$ & $88(5)$ & $1.07(34)$ & $88(4)$ & $0.98(27)$ \\
0.820 & $89(6)$ & $1.25(45)$ & $89(5)$ & $1.20(38)$ & $89(4)$ & $1.07(29)$ \\
0.825 & $88(7)$ & $1.13(50)$ & $88(6)$ & $1.06(40)$ & $88(5)$ & $1.01(32)$ \\
0.830 & $91(6)$ & $1.47(60)$ & $90(5)$ & $1.34(49)$ & $90(4)$ & $1.20(38)$ \\
0.835 & $91(6)$ & $1.49(76)$ & $89(6)$ & $1.18(56)$ & $89(5)$ & $1.07(43)$ \\
0.840 & $95(4)$ & $2.49(1.25)$ & $92(5)$ & $1.66(78)$ & $91(4)$ & $1.37(54)$ \\
0.845 & $89(10)$ & $1.13(95)$ & $86(11)$ & $0.82(65)$ & $84(11)$ & $0.70(51)$ \\
0.850 & $91(8)$ & $1.45(1.20)$ & $87(9)$ & $0.94(73)$ & $85(10)$ & $0.76(55)$ \\
\hline
\end{tabular}

(ii) There are nonanalytic corrections related to the irrelevant operators. They have the form $\left|\beta-\beta_{c}\right|^{n \nu \omega_{i}},\left|\beta-\beta_{c}\right|^{n \nu \omega_{i}+m \nu \omega_{j}}$, etc., where $\omega_{i}=-y_{i}$ and $y_{i}$ are the RG dimensions of the irrelevant $\mathrm{RG}$ operators, and $n, m$ are integers.

(iii) There are corrections related to the analytic background. For instance, in the susceptibility there are corrections proportional to $\left|\beta-\beta_{c}\right|^{\gamma}$.

In [47] an argument was given to show that renormalized coupling constants, like $G_{4}$ and $G_{22}$, do not have analytic corrections. The absence of this type of corrections was verified in the continuum $O(N) \phi^{4}$ theory in $d$ dimensions to order $1 / N$ [47] and in the two-dimensional Ising model [48, 49]. In the absence of analytic corrections, the leading contribution is due to the irrelevant operators, and therefore $\Delta=\omega_{1} \nu$, where $\omega_{1}=-y_{1}$ and $y_{1}$ is the RG dimension of the leading irrelevant operator in the model. Therefore, in Eq. (18) the exponent $\Omega$ should be identified with $\omega_{1}$.

Corrections appearing in Eq. (21) are strictly related to corrections appearing in FSS. In the FSS case no analytic corrections are expected, not only in $G_{4}$ or $G_{22}$ but in any quantity.\| Corrections of type (ii) correspond to terms of the form $L^{-n \omega_{i}}, L^{-n \omega_{i}-m \omega_{j}}$, so that the leading correction in FSS has the form $L^{-\omega_{1}}$. Thus, the exponent $\Omega$ should be identified with the exponent that controls FSS corrections and has been used in the FSS Ansatz (15). The results reported in Tables 3 and 4 give estimates of $\Omega$ that show a tiny dependence on $\omega$ and we can safely estimate

$$
\Omega=1.0(4) \text {. }
$$

\| A careful discussion is presented in [44. The absence of analytic corrections in FSS is shown in 50] and has been checked to order $L^{-2}$ in the two-dimensional Ising model 49] and to order $L^{-1}$ in the three-dimensional XY model [51]. 
Table 4. Estimates of $G_{22}^{*}$ and $\Omega$ for different values of $\omega$. Here $L_{\min }=12$.

\begin{tabular}{llccccc}
\hline & \multicolumn{2}{c}{$\omega=0.2$} & \multicolumn{2}{c}{$\omega=1$} & \multicolumn{2}{c}{$\omega=2$} \\
$\beta_{\min }$ & $G_{22}^{*}$ & $\Omega$ & $G_{22}^{*}$ & $\Omega$ & $G_{22}^{*}$ & $\Omega$ \\
\hline 0.780 & $-8.1(3.8)$ & $0.69(22)$ & $-10.6(1.8)$ & $0.81(16)$ & $-10.1(1.5)$ & $0.76(13)$ \\
0.790 & $-10.2(3.0)$ & $0.88(27)$ & $-11.5(1.6)$ & $0.94(19)$ & $-10.9(1.3)$ & $0.88(16)$ \\
0.800 & $-11.1(2.8)$ & $1.00(32)$ & $-11.8(1.5)$ & $1.01(22)$ & $-11.3(1.3)$ & $0.94(18)$ \\
0.810 & $-10.6(3.3)$ & $0.92(34)$ & $-11.7(1.6)$ & $0.99(24)$ & $-11.1(1.4)$ & $0.90(19)$ \\
0.815 & $-10.1(3.8)$ & $0.86(37)$ & $-11.8(1.7)$ & $1.01(27)$ & $-10.9(1.6)$ & $0.87(21)$ \\
0.820 & $-11.6(2.9)$ & $1.10(44)$ & $-12.3(1.5)$ & $1.14(29)$ & $-11.6(1.3)$ & $1.01(24)$ \\
0.825 & $-9.1(5.4)$ & $0.73(44)$ & $-11.5(2.0)$ & $0.93(30)$ & $-10.2(2.1)$ & $0.75(24)$ \\
0.830 & $-11.2(3.6)$ & $1.03(54)$ & $-12.4(1.6)$ & $1.18(37)$ & $-10.9(1.8)$ & $0.87(27)$ \\
0.835 & $-9.6(5.6)$ & $0.78(55)$ & $-11.5(2.3)$ & $0.91(38)$ & $-10.0(2.7)$ & $0.71(30)$ \\
0.840 & $-12.8(2.9)$ & $1.53(93)$ & $-12.6(1.7)$ & $1.25(49)$ & $-11.1(2.0)$ & $0.90(35)$ \\
0.845 & $-9.5(7.2)$ & $0.73(71)$ & $-10.2(4.0)$ & $0.67(45)$ & $-7.9(5.7)$ & $0.49(36)$ \\
0.850 & $-10.0(6.6)$ & $0.81(80)$ & $-10.3(4.0)$ & $0.68(47)$ & $-8.1(5.7)$ & $0.51(38)$ \\
\hline
\end{tabular}

This result justifies a posteriori our choice of not considering values of $\omega$ smaller than 0.2. The estimate of $\Omega$ restricts the interval in which $\omega$ can vary. This allows us to obtain more precise estimates for $\eta_{o}$ and $G_{22}^{*}$ (the estimate of $G_{4}^{*}$ does not vary since this quantity has a very small dependence on $\omega$ ). By assuming $0.6 \leq \omega \leq 1.4$ we obtain the results:

$$
\begin{aligned}
& \eta_{o}=-0.24(4), \\
& G_{22}^{*}=-11.5(2.5) .
\end{aligned}
$$

Finally, we try to determine the position of the critical point. For this purpose, we analyze $\chi_{\infty}$ and $\xi_{\infty}$ separately as

$$
\begin{gathered}
\log \chi_{\infty}(\beta)=A_{\chi}-\gamma_{o} \log \left(\beta_{c}-\beta\right), \\
\log \xi_{\infty}(\beta)=A_{\xi}-\nu_{o} \log \left(\beta_{c}-\beta\right),
\end{gathered}
$$

where $A_{\chi}, A_{\xi}, \gamma_{o}, \nu_{o}$, and $\beta_{c}$ are free parameters. As before, we repeat the fit several times, including each time only the data with $\beta \geq \beta_{\min }$. Results using the infinitevolume data obtained with $\omega=1$ are reported in Table 5 . The estimates of $\beta_{c}$ show a tiny dependence on $\beta_{\min }$; moreover, the results obtained by using $\chi_{\infty}$ and $\xi_{\infty}$ are fully consistent. We take as our final estimate

$$
\beta_{c}=1.08 \pm 0.01 \pm 0.03
$$

where the first error is statistical and the second one is systematic and takes into account the change in the estimate as $\omega$ varies between 0.6 and 1.4. The data indicate therefore a transition point that is just beyond the interval of $\beta$ where we have made the simulations and thus they are compatible with the absence of a crossing point in $B_{q}$ and $\xi / L$ and with the fact that the estimates of these two quantities at fixed $L$ get closer as $\beta$ increases. 
Table 5. Estimates of $\beta_{c}$ and $\gamma$ (second and third column) obtained from the fit of $\chi_{\infty}$ and of $\beta_{c}$ and $\nu$ (fourth and fifth column) obtained from the fit of $\xi_{\infty}$. The infinitevolume estimates of $\chi_{\infty}$ and $\xi_{\infty}$ have been obtained by applying the extrapolation method based on Eq. (15) with $\omega=1$ to all data $\left(L_{\min }=8\right)$.

\begin{tabular}{clccc}
\hline & \multicolumn{2}{c}{ fit of $\chi_{\infty}$} & \multicolumn{2}{c}{ fit of $\xi_{\infty}$} \\
$\beta_{\min }$ & \multicolumn{1}{c}{$\beta_{c}$} & \multicolumn{1}{c}{$\gamma_{o}$} & \multicolumn{1}{c}{$\beta_{c}$} & \multicolumn{1}{c}{$\nu_{o}$} \\
\hline 0.76 & $1.060(2)$ & $4.78(4)$ & $1.083(3)$ & $2.43(4)$ \\
0.78 & $1.059(2)$ & $4.76(5)$ & $1.075(4)$ & $2.33(4)$ \\
0.80 & $1.060(2)$ & $4.76(6)$ & $1.073(4)$ & $2.29(5)$ \\
0.82 & $1.062(3)$ & $4.84(8)$ & $1.073(5)$ & $2.30(6)$ \\
0.84 & $1.064(4)$ & $4.89(12)$ & $1.072(6)$ & $2.28(8)$ \\
0.86 & $1.070(6)$ & $5.11(19)$ & $1.076(9)$ & $2.35(13)$ \\
0.88 & $1.074(9)$ & $5.27(32)$ & $1.080(13)$ & $2.42(21)$ \\
0.90 & $1.082(16)$ & $5.60(62)$ & $1.080(19)$ & $2.41(34)$ \\
\hline
\end{tabular}

We can also perform a more quantitative check by using the results of [30] for the critical-point values $B_{q}^{*}$ and $(\xi / L)^{*}$. They quote: $B_{q}^{*}=1.475(6)$ (bimodal distribution) and $B_{q}^{*}=1.480(14)$ (Gaussian distribution); $(\xi / L)^{*}=0.627(4)$ (bimodal distribution) and $(\xi / L)^{*}=0.635(9)$ (Gaussian distribution). These results are compatible with ours for $B_{q}$ and $\xi / L$ close to $\beta=1.08$. For $\beta=1.07$ we have $B_{q}=1.411(4)(L=12)$, $B_{q}=1.434(6)(L=16)$, and $\xi / L=0.662(4)(L=12), \xi / L=0.648(4)(L=16)$. These results are very close to the ISGM estimates and show the correct trend. They are therefore consistent with the conjecture that the ISGM and the SRAM belong to the same universality class. Magnetic variables behave differently: as is clear in Fig. 5 . the estimates at fixed $L$ are well separated, so that we do not expect any crossing at $\beta \approx 1.08$. Thus, no criticality is expected in magnetic quantities: below the transition temperature the system is still paramagnetic.

Using the results reported in Table 5, we can also estimate the critical exponents $\gamma_{o}$ and $\nu_{o}$. We obtain:

$$
\begin{aligned}
& \nu_{o}=2.4 \pm 0.2 \pm 0.4 \\
& \gamma_{o}=5.3 \pm 0.3 \pm 1.0 .
\end{aligned}
$$

The estimates correspond to $\beta_{\min }=0.88$. The first reported error is the statistical error and should be large enough to include most of the dependence on $\beta_{\min }$. The second one gives the variation of the estimate with $\omega$.

\section{Conclusions}

In this paper we study the SRAM by MC simulations with the purpose of clarifying whether this model shows a critical behavior. We find clear evidence of FSS and 
criticality, with a critical point located at

$$
\beta_{c}=1.08(4) .
$$

Correspondingly, we compute the critical exponents associated with the critical behavior of overlap observables. We obtain:

$$
\begin{aligned}
& \eta_{o}=-0.24(4) \\
& \nu_{o}=2.4(6) \\
& \gamma_{o}=5.3(1.3) .
\end{aligned}
$$

These estimates are close to those obtained for the ISGM. Ref. 28] quotes $\nu_{o}=2.22(15)$, $\eta_{o}=-0.349(18)$, while [30] reports $\nu_{o}=2.39(5)$ and $\eta_{o}=-0.395(17)$. Other estimates are reported in Table 1 of [30]. With the quoted error bars there is a small discrepancy between our estimate of $\eta_{o}$ and those of [28, 30]. This difference should not be taken too seriously, since there are similar discrepancies among the estimates obtained by different groups for the bimodal ISGM, see Table 1 of 30. For instance, our result is close to $\eta_{o}=-0.26(4)$ reported in [26]. Note that [28] also reports an estimate of the subleading exponent $\omega=0.7(3)$. This result is obtained from fits of $\chi_{\infty}$ vs $\xi_{\infty}$. In this case, however, analytic corrections are expected and therefore the leading scaling correction is $\xi_{\infty}^{-1 / \nu_{o}} \approx \xi_{\infty}^{-0.45}$. Therefore, the result obtained in [28] provides only a strong indication that $\omega \gtrsim 1 / \nu_{o}$ but does not give a quantitative estimate of the exponent associated with the leading irrelevant operator. The bound $\omega \gtrsim 1 / \nu_{o}$ is fully consistent with our results.

The possibility that the SRAM is in the same universality class of the ISGM was put forward in 14] and found to be consistent with numerical results in two dimensions in [31. It looks very plausible, since the SRAM is nothing but an Ising model with local disorder and frustration. In some sense we can think of the SRAM and of the ISGM as two different versions of the same model: in the SRAM disorder is associated with lattice sites, while in the ISGM disorder is associated with lattice bonds. They are analogous to the site-diluted and bond-diluted Ising model, whose Hamiltonian is given by Eq. (3) with $j_{x y}=r_{x} r_{y}$ (site dilution) and $j_{x y}=r_{x y}$ (bond dilution), $r$ being a random variable such that $r=1(r=0)$ with probability $p$ (resp. $1-p)$. Note that the site-diluted model can also be seen as a bond-diluted model with a correlated bond distribution, exactly as is the case for the SRAM. Nonetheless, there is little doubt - though a precise numerical check is still missing - that the two models belong to the same universality class.

Note that the SRAM is less frustrated than the standard ISGM, since in the SRAM $\overline{\prod_{\square} j_{x y}}=1 / 27$. This fact does not rule out our conjecture since it is known that maximal frustration is not necessary to obtain glassy behavior. For instance, the random-bond Ising model with $j_{x y}=+1$ with probability $p$ and $j_{x y}=-1$ with probability $1-p$ has a glassy low-temperature phase for [52] $0.233 \lesssim p \lesssim 0.767$.

It is interesting to generalize to SRAM by considering $N$-dimensional unit vectors $\vec{u}_{i}$. The correlation of the bond variables around a lattice plaquette becomes $\overline{\prod_{\square} j_{x y}}=$ $1 / N^{3}$, which implies that bond correlations vanish for $N \rightarrow \infty$. Thus, for $N=\infty$, the 
SRAM is just an ISGM with a different continuous bond distribution. In this limit, therefore, the two models belong to the same universality class. If our conjecture for $N=3$ is correct, the same should hold for any $N \geq 3$. For $N=1$ it is enough to redefine $\sigma_{i} \rightarrow u_{i} \sigma_{i}$ to reobtain the standard ferromagnetic Ising model. The behavior for $N=2$ is unclear, since this model is less frustrated than that with $N=3$ studied here. In analogy with what happens in the random-bond Ising model mentioned in the previous paragraph, it could have a glassy transition or a ferromagnetic transition (the nature of the ferromagnetic transition is still object of debate, see [53]).

Our results are not precise enough to show conclusively that the SRAM and the ISGM belong to the same universality class. Because of the somewhat large scaling corrections, one needs to perform simulations on larger lattices and deeper in the critical regime (note that for $L=30$, our largest lattice, our data extend only up to $\beta=0.95$, that is quite far from the critical point) to obtain precise and reliable estimates of the critical exponents. In particular, it would be interesting to see whether the small difference in the estimates of $\eta$ disappears (at the same time it is important to include scaling corrections in the analysis of the ISGM results, to understand the reliability of the quoted error bars). Since we have reasonably precise estimates for $G_{4}^{*}$ and $G_{22}^{*}$, it is also interesting to compute the same quantities in the ISGM. This would provide an additional check of the conjecture. Work in this direction is in progress.

A question that remains open is the behavior of the RAM for finite anisotropy $D$. If there is indeed a low-temperature phase with QLRO for small $D$ as predicted in [11, 12, 13], then there should be a critical value $D^{*}$ such that ISGM behavior is observed only for $D>D^{*}$. Nothing is known about $D^{*}$ and we cannot even exclude that $D^{*}=\infty$, so that ISGM behavior is observed only for model (3).

\section{References}

[1] Harris R, Plischke M and Zuckermann M J, New model for amorphous magnetism, 1973 Phys. Rev. Lett. 31, 160.

[2] Calabrese P, Pelissetto A and Vicari E, Spin models with random anisotropy and reflection symmetry, 2004 Phys. Rev. E 70, 036104 cond-mat/0311576.

[3] Campostrini M, Hasenbusch M, Pelissetto A, Rossi P and Vicari E, Critical exponents and equation of state of the three-dimensional Heisenberg universality class, 2002 Phys. Rev. B 65, 144520 cond-mat/0110336.

[4] Pelissetto A and Vicari E, Critical phenomena and renormalization-group theory, 2002 Phys. Rep. 368, 549 cond-mat/0012164.

[5] Dudka M, Folk R and Holovatch Yu, Critical properties of random anisotropy magnets, $2005 \mathrm{~J}$. Magn. Magn. Mater. 294, 305 cond-mat/0406692.

[6] Imry Y and Ma S-k, Random-field instability of the ordered state of continuous symmetry, 1975 Phys. Rev. Lett. 35, 1399.

[7] Pelcovits R A, Pytte E and Rudnick J, Spin-glass and ferromagnetic behavior induced by random uniaxial anisotropy, 1978 Phys. Rev. Lett. 40, 476; Erratum 1982 48, 1297.

[8] Aharony A, Critical behavior of amorphous magnets, 1975 Phys. Rev. B 12, 1038.

[9] Mukamel D and Grinstein G, Critical behavior of random systems, 1982 Phys. Rev. B 25, 381.

[10] Dudka M, Folk R and Holovatch Yu, On the critical behaviour of random anisotropy magnets, 2001 Cond. Matt. Phys. 4, 77; Dudka M, Folk R and Holovatch Yu, 2001 Phase Transition in 
the Random Anisotropy Model, in Fluctuating Paths and Fields, edited by Janke W, Pelster A, Schmidt H-J and Bachmann M (Singapore: World Scientific) cond-mat/0106334.

[11] Aharony A and Pytte E, Infinite susceptibility phase in random uniaxial anisotropy magnets, 1980 Phys. Rev. Lett. 45, 1583; Low-temperature scaling for systems with random fields and anisotropies 1983 Phys. Rev. B 27, 5872.

[12] Feldman D E, Quasi-long-range order in the random anisotropy Heisenberg model: Functional renormalization group in $4-\epsilon$ dimensions, 2000 Phys. Rev. B 61, 382.

[13] Feldman D E, Quasi-long range order in glass states of impure liquid crystals, magnets, and superconductors, 2001 Int. J. Mod. Phys. B 15, 2945 cond-mat/0201243.

[14] Chen J H and Lubensky T C, Mean field and $\epsilon$-expansion of spin glasses, 1977 Phys. Rev. B 16, 2106.

[15] Mézard M, Parisi G and Virasoro M A, 1987 Spin-Glass Theory and Beyond (Singapore: World Scientific).

[16] Fischer K H and Hertz J A, 1991 Spin Glasses (Cambridge: Cambridge University Press).

[17] Kawashima N and Rieger H, 2004 Recent progress in spin glasses in Frustrated Spin Systems, edited by Diep H T (Singapore: World Scientific) cond-mat/0312432.

[18] Jayaprakash C and Kirkpatrick S, Random anisotropy models in the Ising limit, 1980 Phys. Rev. B 21, 4072 .

[19] Chakrabarti A, Spin-glass transition in three-dimensional random-anisotropy-axis model, 1987 Phys. Rev. B 36, 5747.

[20] Fisch R, Low-temperature behavior of random-anisotropy magnets, 1990 Phys. Rev. B 42, 540.

[21] Fisch R, Quasi-long-range order in random-anisotropy Heisenberg models, 1998 Phys. Rev. B 58, 5684 cond-mat/9801033.

[22] Itakura M, Frozen quasi-long-range order in the random anisotropy Heisenberg magnet, 2003 Phys. Rev. B 68, 100405(R) cond-mat/0303552.

[23] Holovatch Yu, Blavats'ka V, Dudka M, von Ferber C, Folk R and Yavors'kii T, Weak quenched disorder and criticality: resummation of asymptotic(?) series, 2003 Int. J. Mod. Phys. B 16, 4027 cond-mat/0111158.

[24] Folk R, Holovatch Yu and Yavors'kii T, Critical exponents of a three dimensional weakly diluted quenched Ising model, 2003 Physics Uspekhi 46, 169 cond-mat/0106468.

[25] Caracciolo S, Edwards R G, Ferreira S J, Pelissetto A and Sokal A D, Extrapolating Monte Carlo Simulations to Infinite Volume: Finite-Size Scaling at $\xi / L \gg$ 1, 1995 Phys. Rev. Lett. 74, 2969 hep-lat/9409004.

[26] Palassini M and Caracciolo S, Universal finite-size scaling functions in the 3D Ising spin glass, 1999 Phys. Rev. Lett. 82, 5128 cond-mat/9904246.

[27] Calabrese P, Martín-Mayor V, Pelissetto A and Vicari E, Three-dimensional randomly dilute Ising model: Monte Carlo results, 2003 Phys. Rev. E 68, 036136 cond-mat/0306272.

[28] Jörg T, Critical behavior of the three-dimensional bond-diluted Ising spin glass: Finite-size scaling functions and universality, 2006 cond-mat/0602215

[29] Jörg T, Lukic J, Marinari E and Martin O C, Strong universality and algebraic scaling in twodimensional Ising spin glasses, 2006 cond-mat/0601480

[30] Katzgraber H, Körner M and Young A P, Detailed study of universality in three-dimensional Ising spin glasses, 2006 cond-mat/0602212

[31] Bray A J and Moore M A, Evidence for spin-glass behaviour in the random anisotropy-axis model, 1985 J. Phys. C: Solid State 18, L139.

[32] Houdayer J, A cluster Monte Carlo algorithm for 2-dimensional spin glasses, 2001 Eur. Phys. J. B 22, 479 cond-mat/0101116.

[33] Wang J-S and Swendsen R H, Replica Monte Carlo simulation (Revisited), Proceedings of the conference Statistical Physics of Disordered Systems and Its Applications, Hayama (Japan), July 2004, 2005 Progr. Theor. Phys. Suppl. 157, 317 cond-mat/0407273.

[34] Jörg T, Cluster Monte Carlo algorithms for diluted spin glasses, Proceedings of the conference 
Statistical Physics of Disordered Systems and Its Applications, Hayama (Japan), July 2004, 2005 Progr. Theor. Phys. Suppl. 157, 349 cond-mat/0410328.

[35] Geyer C J, 1991 Markov chain Monte Carlo maximum likelihood, in Computer Science and Statistics: Proc. of the 23rd Symposium on the Interface, edited by E. M. Keramidas (Fairfax Station: Interface Foundation), p. 156.

[36] Geyer C J and Thompson E A, Annealing Markov-chain Monte-Carlo with applications to ancestral inference, $1995 \mathrm{~J}$. Am. Statist. Association 90, 909.

[37] Hukushima K and Nemoto K, Exchange Monte Carlo method and application to spin glass simulations, 1996 J. Phys. Soc. Japan 65, 1604 cond-mat/9512035.

[38] Earl D J and Deem M W, Parallel tempering: theory, applications, and new perspectives, 2005 Phys. Chem. Chem. Phys. 7, 3910 physics/0508111.

[39] Katzgraber H G, Trebst S, Huse D A and Troyer M, Feedback-optimized parallel tempering Monte Carlo, 2006 J. Stat. Mech.: Theory Expt., P03018 cond-mat/0602085.

[40] Caracciolo S, Edwards R G, Pelissetto A and Sokal A D, Asymptotic Scaling in the TwoDimensional $O(3) \sigma$ model at correlation length $10^{5}$, 1995 Phys. Rev. Lett. 75, 1891 hep-lat/9411009.

[41] Mana G, Pelissetto A and Sokal A D, Multigrid Monte Carlo simulation via XY embedding. II. Two-dimensional SU(3) principal chiral model, 1997 Phys. Rev. D 55, 3674 hep-lat/9610021.

[42] Caracciolo S and Pelissetto A, Corrections to finite-size scaling in the lattice $N$-vector model for $N=\infty, 1998$ Phys. Rev. D 58, 105007 hep-lat/9804001.

[43] Privman V (ed.), 1990 Finite Size Scaling and Numerical Simulations of Statistical Systems (Singapore: World Scientific).

[44] Salas J and Sokal A D, Universal amplitude ratios in the critical two-dimensional Ising model on a torus, 2000 J. Statist. Phys. 98, 551 cond-mat/9904038.

[45] Wegner F J, 1976 in Phase Transitions and Critical Phenomena, Vol. 6, edited by Domb C and Green M S (New York: Academic) p. 7.

[46] Aharony A and Fisher M E, Nonlinear scaling fields and corrections to scaling near criticality, 1982 Phys. Rev. B 27, 4394.

[47] Pelissetto A and Vicari E, Four-point renormalized coupling constant and Callan-Symanzik $\beta$ function in $O(N)$ models, 1998 Nucl. Phys. B 519, 626 cond-mat/9711078.

[48] Calabrese P, Caselle M, Celi A, Pelissetto A and Vicari E, Non-analyticity of the CallanSymanzik $\beta$-function of two-dimensional $O(N)$ models, 2000 J. Phys. A: Math. Gen. 33, 8155 hep-th/0005254.

[49] Caselle M, Hasenbusch M, Pelissetto A and Vicari E, Irrelevant operators in the two-dimensional Ising model, 2002 J. Phys. A: Math. Gen. 35, 4861 cond-mat/0106372.

[50] Guo H and Jasnow D, Hyperuniversality and the renormalization group for finite systems, 1987 Phys. Rev. B 35, 1846.

[51] Campostrini M, Hasenbusch M, Pelissetto A and Vicari E, The critical exponents of the superfluid transition in ${ }^{4} \mathrm{He}, 2006$ cond-mat/0605083

[52] Ozeki Y and Ito N, Multicritical dynamics for the $\pm J$ Ising model, 1998 J. Phys. A: Math. Gen. 31, 5451 .

[53] Ito N, Ozeki Y and Kikatani N, Non-universal critical behavior in the ferromagnetic transition of the $\pm J$ Ising model, 1999 J. Phys. Soc. Jpn. 68, 803. 